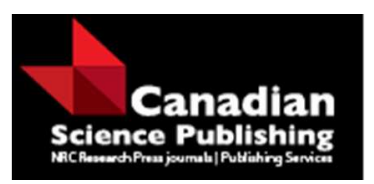

Canadian Journal of Physics

Revue canadienne de physique

\title{
Quantum-thermal self-diffusion as a hydrodynamic mechanism for the fluctuations relaxation
}

\begin{tabular}{|r|l|}
\hline Journal: & Canadian Journal of Physics \\
\hline Manuscript ID & cjp-2014-0402.R1 \\
\hline Danuscript Type: & Article \\
\hline Complete List of Authors: & $\begin{array}{l}\text { Golubjeva, OLGA; Peoples' Friendship University of Russia, Institute of } \\
\text { Gravitation and Cosmology } \\
\text { Sidorov, Sergey; Peoples' Friendship University of Russia, Institute of } \\
\text { Gravitation and Cosmology }\end{array}$ \\
\hline Keyword: & $\begin{array}{l}\text { quantum stochastic influence, density of diffusion pressure, self- } \\
\text { diffusion, effective influence, thermal stochastic influence }\end{array}$ \\
\hline & \\
\hline
\end{tabular}

SCHOLARONE $^{\text {Im }}$ 


\title{
Quantum-thermal self-diffusion as a hydrodynamic mechanism for the fluctuations relaxation.
}

\author{
Olga Golubjeva * and Sergey Sidorov *
}

\begin{abstract}
We propose a generalization of quantum mechanical equations in the hydrodynamic form by introducing, into the Lagrangian density, terms taking into account the diffusion velocity at zero and finite temperatures and the diffusion pressure energy of the warm vacuum. Based on this, for the model of one-dimensional hydrodynamics, we construct a system of equations that are analogous to the Euler equations but with the inclusion of quantum and thermal effects. They are a generalization of equations of the Nelson stochastic mechanics. The numerical analysis of the behavior of solutions of this system allows concluding that this system can be used to describe the process of quantum-thermal fluctuation relaxation.

Key words: quantum stochastic influence, thermal stochastic influence, effective influence, self-diffusion, density of diffusion pressure energy, drift and diffusion velocities, fluctuations, numerical analysis.
\end{abstract}

PACS $\{02.60 . D c, 03.65 .-\mathrm{w}\}$

*Peoples' Friendship University of Russia, 117198, Moscow, Russia. E-mail: ogol2013@gmail.com 


\section{Introduction}

Thermal fluctuations in hydrodynamics have been taken into account during the fifty years; however, there is still no consistent quantum statistical theory account for quantum and thermal effect simultaneously [?]. In this paper, we present a variant of the approach to the construction of such a theory, starting from the hydrodynamic form of quantum mechanics. For this purpose. we propose to generalize it considering quantum-thermal diffusion, which reflects the stochastic character of the environmental influence, and the density of diffusion pressure energy, which exists at zero and finite Kelvin temperatures. In this case, it is necessary to use the generalization of the concept of thermal equilibrium to the case of the simultaneous inclusion of stochastic influence of the quantum and thermal types. As a result, for a one-dimensional model, we obtain a system of hydrodynamic equations that is analogous to the system of Euler equations, but differs from it, because it takes quantum and thermal effects into account.

The hydrodynamic equations are traditionally derived either from the statistical mechanics or from the kinetics, which use concrete concepts of medium structure and interactions between its components. Accordingly, hydrodynamic fluctuations are accounted for by including, in the hydrodynamic equations, a random stress tensor (together with the regular one), for which only one correlator is given on the basis of the fluctuationdissipation theorem (FDT).

At the same time, hydrodynamics is similar conceptually to equilibrium thermodynamics, because it is also a modelless theory in essence. Therefore, we propose to consider the theory of resolution of quantum-thermal fluctuations of the density and the drift velocity at equilibrium with respect to the temperature as a stochastic hydrodynamics. In this case, we can begin to derive the corresponding equations by generalizing the hydrodynamic form of quantum mechanics at zero temperature as a modelless theory to the case of the explicit inclusion of self-diffusion in the cold and warm vacuums. This allows, for the first time, extending the hydrodynamic form of quantum mechanics to finite temperatures and considering not only self-diffusion, but also the diffusion pressure of the warm vacuum.

As a result, for the one-dimensional model, we obtain a system of equations of stochastic hydrodynamics that is valid at any temperatures. Its distinction consists in that it takes quantum and thermal fluctuations into account nonadditively. Moreover, we have managed to endow these equations with the form of the equations of two-velocity hydrodynamics, which is a generalization of the Nelson stochastic mechanics.

\section{Effective influence as a universal characteristic of transport processes: Self- diffusion coefficient}

In our study, we rely on the results, which we obtained previously in [?]. In it, we developed a special macroparameter, namely the effective influence $\mathbb{J}$ in the form 


$$
\mathbb{J}=\frac{\hbar}{2} \operatorname{coth} \frac{\varkappa \omega}{T}=\frac{\hbar}{2} \operatorname{coth} \frac{\hbar \omega}{2 k_{B} T} .
$$

that characterized interaction between a system and its environment (thermostat). It plays a significant role in our farther contractions. We note that at $T=0$ this quantity has the minimal value $\hbar / 2$. This fact says that, in this case, a system undergoes only purely quantum effect. In his turn, at some $T \neq 0$ quantity $\mathbb{J}$ depends on the constants Planck and Boltzmann simultaneously. This is a striking illustration of the fact that a system undergoes not only the purely quantum effect but the thermal effect also. In the limit of high temperatures the effect of quantum processes disappears.

Effective transport coefficients ( the diffusion coefficient that is the subject of our interest, first of all), which are typical of nonequilibrium thermodynamics, can also be expressed in terms of this quantity. Their stochastic nature is thus demonstrated. The latter is obviously seen if the self-diffusion process is used as an example. It occurs in the medium with the nonuniform density after the equilibrium with respect to the temperature is established.

Indeed, it has already been shown in the theory of Brownian motion at rather high temperatures [?] that, in this case (for $t \gg \tau$ ), the uncertainty relation of the form

$$
(\Delta p) \cdot(\Delta q)=m D_{T}
$$

is valid. Here, $D_{T}$ is the coefficient of purely thermal diffusion; in particular, $D_{T}=k_{B} T \tau / m$, where $\tau$ is the relaxation time, for a free microparticle, while $D_{T}=k_{B} T / m \omega$ for a Brownian oscillator [?].

As shown in [?], the Schrödinger uncertainty relation "momentum-coordinate" for a quantum oscillator in the state of equilibrium with a thermostat has the form

$$
(\Delta p) \cdot(\Delta q)=\mathbb{J}=\frac{\hbar}{2} \operatorname{coth} \frac{\hbar \omega}{2 k_{B} T} .
$$

Comparing (??) and (??), we rewrite this relation in the form

$$
(\Delta p) \dot{(} \Delta q)=m \mathbb{D} \text {. }
$$

Then it is natural to call the quantity

$$
\mathbb{D}=\frac{\hbar}{2} \operatorname{coth} \frac{\hbar \omega}{2 k_{B} T} \equiv \frac{\mathbb{J}}{m}
$$

the effective self-diffusion coefficient. We note that Nelson [?] called the quantity $\hbar / 2 m$ the quantum diffusion coefficient $\hbar / 2 m=D_{q u}$, i.e., in the case of the contact with the so-called vacuum or, in other words, in the absence of the thermal environmental influence at $T=0$. 
It follows from (??) that the coefficient $\mathbb{D}$ acquires the physical meaning of the effective influence per mass unit. The respective limiting values of $\mathbb{D}$ at high and low Kelvin temperatures are

$$
\begin{gathered}
\mathbb{D} \rightarrow D_{T}=\frac{k_{B} T}{m \omega}, \quad \text { high temperatures } \quad k_{B} T \gg \hbar \omega / 2, \\
\mathbb{D} \rightarrow D_{q u} \equiv \frac{\hbar}{2 m}, \quad \text { low temperatures } \quad k_{B} T \ll \hbar \omega / 2 .
\end{gathered}
$$

Starting from relation (??), one can also introduce the other effective transport coefficients in terms of $\mathbb{J}$, namely, coefficients of heat conductivity, shear viscosity, and others that are important for nonequilibrium processes. Thus, the majority of transport coefficients can be expressed in terms of the effective self-diffusion coefficient $\mathbb{D}$, which can be, in principle, measured in experiments.

As for the constant $\varkappa \equiv \frac{\hbar}{2 k_{B}}$, it can be expressed in terms of observed transport coefficients by means of relations of the following type when analyzing particular experiments:

$$
\varkappa=\left(\frac{\mathbb{D}}{\mathbb{S} / m}\right)_{\min }=\left(\frac{\eta_{e f}}{\mathbb{S} / V}\right)_{\min }=\ldots
$$

where $\mathbb{S} / m$ is the effective entropy of the mass unit, $\mathbb{S} / V$ is the effective entropy of the volume unit, and $\eta_{e f}$ is the effective shear-viscosity coefficient.

\section{Standard quantum mechanics in the hydrodymanic form}

In the nonrelativistic field form, the standard quantum mechanics (at $T=0$ ) can be obtained in the case where the action functional variation becomes zero [?]:

$$
\mathcal{S}=\int_{t_{1}}^{t_{2}} d t \int d q \mathcal{L}_{0}\left[\psi^{*} ; \psi\right]
$$

Here, $\mathcal{L}_{0}\left[\psi^{*} ; \psi\right]$ is the Lagrangian density for one spinless particle at $T=0$, and $\psi(q, t)$ and $\psi^{*}(q, t)$ are the wave function and its complex conjugate function; they have the meaning of independent nonrelativistic fields. We restrict ourselves to the one-dimensional case. form

It is obvious that, in the general case, the functional $\mathcal{L}_{0}\left[\psi^{*} ; \psi\right]$ must be chosen in the

$$
\mathcal{L}_{0}\left[\psi^{*} ; \psi\right]=\psi^{*}(q, t)\left(i \hbar \frac{\partial}{\partial t}+\frac{\hbar^{2}}{2 m} \frac{\partial^{2}}{\partial q^{2}}\right) \psi(q, t)-\psi^{*}(q, t) U(q) \psi(q, t),
$$

where the nonrelativistic limit of the Klein-Gordon operator is in brackets on the right, and the potential energy operator $U(q)$ characterizes the regular influence energy.

The independent variation of the action of form (??) with respect to the field $\psi^{*}$ leads to the condition

$$
\int_{t_{1}}^{t_{2}} d t \int d q \frac{\delta \mathcal{L}_{0}\left[\psi^{*} ; \psi\right]}{\delta \psi}=\int_{t_{1}}^{t_{2}} d t \int d q\left(i \hbar \frac{\partial \psi}{\partial t}+\frac{\hbar^{2}}{2 m} \frac{\partial^{2} \psi}{\partial q^{2}}-U(q) \psi\right)=0
$$


which leads to the Schrödinger equation

$$
i \hbar \frac{\partial \psi}{\partial t}=-\frac{\hbar^{2}}{2 m} \frac{\partial^{2} \psi}{\partial q^{2}}+U(q) \psi
$$

Accordingly, its complex conjugate equation is obtained when varying the action of form (??) with respect to $\psi$ and differs from formula (??) by the replacement of $i$ with $-i$ and of $\psi$ with $\psi^{*}$. We stress that the Schrödinger equations for the complex wave functions $\psi$ and $\psi^{*}$ have t he meaning of the Euler-Lagrange equations; in this case, wave functions are always complex in the full-scale quantum mechanics.

We now represent the wave function in the form

$$
\psi(q, t)=\sqrt{\rho(q, t)} \exp \{i \theta(q, t)\}
$$

where $\rho(q, t)=|\psi(q, t)|^{2}$. It could be possible to substitute this expression, along with its complex conjugate expression, directly in the Schrödinger equations for $\psi$ and $\psi^{*}$ and obtain the system of equations for the functions $\rho(q, t)$ and $\theta(q, t)$ that has long been known in the literature as quantum mechanics in the hydrodynamic form [?], [?].

Because our aim is to construct modified hydrodynamics based on the microdescription, we propose another approach to the problem. It requires to develop the theory in the Lagrange formulation from the beginning. Therefore, we start from transforming the Lagrangian density $\mathcal{L}_{0}$ to variables that are most suited to the hydrodynamic description. As functional arguments of the Lagrangian density, we choose two independent real functions, namely, the probability density $\rho$ and the phase $\theta$ instead of the complex wave functions $\psi$ and $\psi^{*}$. In essence, they are close to the functions of the mass density $\rho_{m}$ and drift velocity $v \sim \frac{\partial \theta}{\partial q}$, which are typical of standard hydrodynamics.

To do this, we replace the arguments in the Lagrangian density (??) by substituting expression (??) and the corresponding expression for $\psi^{*}$ in it. After the substitution, we obtain

$$
\begin{aligned}
\mathcal{L}_{0}\left[\psi ; \psi^{*}\right]=\mathcal{L}_{0}[\rho ; \theta] & =-\hbar \frac{\partial \theta}{\partial t} \rho-\frac{\hbar^{2}}{2 m}\left(\frac{\partial \theta}{\partial q}\right)^{2} \rho-\frac{\hbar^{2}}{8 m}\left(\frac{\partial \rho}{\partial q}\right)^{2} \frac{1}{\rho}- \\
& -U(q) \rho+i \frac{\hbar}{2} \frac{\partial \rho}{\partial t}+\frac{\hbar^{2}}{2 m} \cdot \frac{\partial}{\partial q}\left(\frac{1}{2} \frac{\partial \rho}{\partial q}+i \rho \frac{\partial \theta}{\partial q}\right) .
\end{aligned}
$$

Here, the term containing $\frac{\partial \rho}{\partial t}$ cannot be taken into account, because it gives the zero contribution when varying the action $\mathcal{S}$ of form (??) with respect to both $\theta$ and $\rho$ in what follows. The last term in (??) is the total derivative with respect to $q$, so that it can also be excluded from the definition of $\mathcal{L}_{0}[\rho ; \theta]$. Therefore, as an expression for the Lagrangian density $\mathcal{L}_{0}[\rho ; \theta]$, we finally take the following expression:

$$
\mathcal{L}_{0}[\rho ; \theta]=-\hbar \frac{\partial \theta}{\partial t} \rho-\frac{\hbar^{2}}{2 m}\left(\frac{\partial \theta}{\partial q}\right)^{2} \rho-\frac{\hbar^{2}}{8 m}\left(\frac{\partial \rho}{\partial q}\right)^{2} \frac{1}{\rho}-U(q) \rho .
$$


Consistently varying the action $\mathcal{S}$ of form (??), in which now $\mathcal{L}_{0}[\rho ; \theta]$ has form (??), with respect to the variable $\theta$ and $\rho$, we obtain the equations for the real functions $\rho(q, t)$ and $\theta(q, t)$ :

$$
\begin{aligned}
& \frac{\partial \rho}{\partial t}+\frac{\partial}{\partial q}\left(\rho \frac{\hbar}{m} \frac{\partial \theta}{\partial q}\right)=0 \\
& \hbar \frac{\partial \theta}{\partial t}+\frac{\hbar^{2}}{2 m}\left(\frac{\partial \theta}{\partial q}\right)^{2}+U(q)-\frac{\hbar^{2}}{8 m}\left[\frac{1}{\rho^{2}}\left(\frac{\partial \rho}{\partial q}\right)^{2}+2 \frac{\partial}{\partial q}\left(\frac{1}{\rho} \frac{\partial \rho}{\partial q}\right)\right]=0 .
\end{aligned}
$$

These equations coincide with the equations that could be obtained for the functions $\rho$ and $\theta$ directly from the Schrödinger equations. However, it is now clear that they have the meaning of the Lagrange-Euler equations for the action $\mathcal{S}$ of form (??) expressed in terms of the variables $\rho$ and $\theta$.

It is assumed traditionally that Eq. (??) is the continuity equation for $\rho(q, t)$. In turn, Eq. (??) is an analogue of the Hamilton-Jacobi equation if the fact that the quantity $\hbar \theta(q, t)$ has the dimensionality of action is taken into account. In this case, the term in brackets in formula (??) is sometimes treated as an additional energy of quantum nature $U_{q u}(q)$ vanishing in the quasiclassical limit as $\hbar \rightarrow 0$.

Of course, Eqs. (??) and (??) for $\rho$ and $\theta$ and the Schrödinger equations for $\psi$ and $\psi^{*}$ are equivalent formally. However, the derivation of the quantum mechanical equations in hydrodynamic form (??) and (??) directly from the principle of least action is physically more preferable to construct stochastic hydrodynamics. At the same time, to obtain the desired result, we must solve the problem of the form of the Lagrangian density (which remains unsolved), in which, in our opinion, the stochastic influence of the environment (the quantum thermostat) must be consistently taken into account.

\section{Quantum self-diffusion at the temperature $T=0$}

To reveal the possibility of generalizing $\mathcal{L}_{0}[\rho ; \theta]$, we first consider the case of the temperature $T=0$ (so-called "the cold vacuum"). To do this, we endow the second and third terms on the right in expression (??) with the physical meaning. In accordance with the terminology introduced by Kolmogorov [?] for Markovian processes in the general theory of stochastic processes and used by Nelson [?] in its stochastic mechanics, we call the quantity

$$
v \equiv \frac{\hbar}{m} \frac{\partial \theta}{\partial q}
$$

the drift velocity. Accordingly, we call the quantity

$$
u \equiv-D_{q u} \frac{1}{\rho} \frac{\partial \rho}{\partial q}=-\frac{\hbar}{2 m} \frac{1}{\rho} \frac{\partial \rho}{\partial q}
$$

the diffusion velocity in the "cold vacuum" and stress its stochastic quantum nature initially. 
Using the velocities $v$ and $u$, we can write formulas (??) -(??) in the form

$$
\begin{gathered}
\mathcal{L}_{0}[\rho, \theta]=-\hbar \frac{\partial \theta}{\partial t} \rho-\frac{m}{2}\left(v^{2}+u^{2}\right) \rho-U \rho, \\
\frac{\partial \rho}{\partial t}+\frac{\partial}{\partial q}(\rho v)=0 \\
\hbar \frac{\partial \theta}{\partial t}+\frac{m}{2} v^{2}+U-\frac{m}{2}\left[u^{2}-\frac{\hbar}{m} \frac{\partial u}{\partial q}\right]=0,
\end{gathered}
$$

providing a possibility of generalizing the Lagrangian density.

It follows from formula (22a) that standard continuity equation (22) is of quasiclassical character, because the probability flux density in it depends only on the drift velocity $v$, while the diffusion velocity $u$ generated by the stochastic influence of the "cold vacuum" is not taken into account in it.

In connection with this, we recall that the Fokker-Planck equation

$$
\frac{\partial \rho}{\partial t}+\frac{\partial}{\partial q}(\rho V)=0
$$

which contains the total velocity of the probability flux density

$$
V=v+u
$$

is the most general continuity equation in accordance with Kolmogorov [?]. We show that it allows describing the approximation to the thermal equilibrium state because of self-diffusion, including the case of the "cold vacuum".

Attention is drawn to the fact that the combination $\frac{m}{2}\left(v^{2}+u^{2}\right)$ contained in expression (21) for $\mathcal{L}_{0}[\rho, \theta]$ is the sum of independent contributions of the kinetic energies of the drift and diffusion motions. At the same time, the probability flux depends on the total velocity of form (??). In connection with this, to obtain the Fokker-Planck equation, in expression (21), the natural replacement of $\left(v^{2}+u^{2}\right)$ with $V^{2}$ must be performed, which allows taking into account the total expression for the kinetic energy related to the probability flux. Thus, even the standard quantum mechanics (at $T=0$ ) admits the possibility to generalize.

Thus, we generalize the Lagrangian density $\mathcal{L}_{0}[\rho, \theta]$ of form (21a) by means of the corresponding replacement. Then we obtain

$$
\tilde{\mathcal{L}}_{0}[\rho, \theta]=-\hbar \frac{\partial \theta}{\partial t} \rho-\frac{m}{2} V^{2} \rho-U \rho=\mathcal{L}_{0}[\rho ; \theta]-m v u \rho=\mathcal{L}_{0}[\rho ; \theta]+\frac{\hbar^{2}}{2 m} \frac{\partial \theta}{\partial q} \frac{\partial \rho}{\partial q} .
$$

Varying the action functional $\mathcal{S}$ of form (??) with $\tilde{\mathcal{L}}_{0}[\rho, \theta]$ with respect to $\theta$ automatically leads to the Fokker-Planck equation with the quantum diffusion coefficient $D_{q u}$

$$
\frac{\partial \rho}{\partial t}+\frac{\partial}{\partial q}(\rho V)=\frac{\partial \rho}{\partial t}+\frac{\partial}{\partial q}\left(\rho \frac{\hbar}{m} \frac{\partial \theta}{\partial q}\right)-D_{q u} \frac{\partial^{2} \rho}{\partial q^{2}}=0 .
$$


At the same time, varying $\mathcal{S}$ with respect to $\rho$ barely changes the Hamilton-Jacobi equation, in which the additional insignificant term appears in comparison with (23a). As a result, the analogue of Eq. (23a) becomes

$$
\hbar \frac{\partial \theta}{\partial t}+\frac{m}{2} v^{2}+U-\frac{m}{2}\left(u^{2}-\frac{\hbar}{m} \frac{\partial u}{\partial q}\right)+\frac{\hbar}{2} \frac{\partial v}{\partial q}=0 .
$$

Obtained equations (??) and (??) generalize Eqs. (22a) and (23a), which allows consistently taking the quantum stochastic influence of the "cold vacuum" into account.

\section{Self-diffusion in the thermostat at the temperature $T \neq 0$}

We now use the approach developed above to the description of self-diffusion simultaneously taking quantum and thermal effect into account. To do this, we introduce the temperature-dependent Lagrangian density $\tilde{\mathcal{L}}_{T}[\rho, \theta]$ and require that it transform into the expression $\tilde{\mathcal{L}}_{0}[\rho, \theta]$ of form (??) as $T \rightarrow 0$. To do this, it suffices to replace the diffusion coefficient $D_{q u}$ with $\mathbb{D}$ of form (??) in expression (??) for the diffusion velocity and introduce the additional term $U_{T}(q) \rho$. The latter takes into account the density of the diffusion pressure energy because of the thermal stochastic environmental influence in the expression for the Lagrangian density.

In our opinion, the expression for $U_{T}$ must have the form that is analogous to the factor $-m u^{2} / 2$ in the cold vacuum (21). However, it must be modified so that $U_{T} \rightarrow 0$ as $T \rightarrow 0$. We introduce it as follows:

$$
U_{T}(q)=-\frac{m}{2}\left[\frac{\alpha}{\Upsilon}\right]^{2} u_{e f}^{2}=-\frac{\hbar^{2}}{8 m} \alpha^{2}\left(\frac{1}{\rho} \frac{\partial \rho}{\partial q}\right)^{2},
$$

where the notations $\alpha^{2} \equiv \sinh ^{-2} \frac{\varkappa \omega}{T} ; \quad \Upsilon=\operatorname{coth} \frac{\varkappa \omega}{T}$ and $u_{e f} \equiv-\mathbb{D} \frac{1}{\rho} \frac{\partial \rho}{\partial q} \quad$ are used. Here $u_{e f}$ is the effective diffusion velocity in the warm vacuum. This quantity is defined analogously to the velocity $u$ in (??), but it is now expressed in terms of the effective diffusion coefficient $\mathbb{D}$ of form (??).

Thus, as the Lagrangian density at $T \neq 0$, we choose the expression

$$
\tilde{\mathcal{L}}_{T}(\rho, \theta)=-\hbar \frac{\partial \theta}{\partial t} \rho-\frac{m}{2}\left(v+u_{e f}\right)^{2} \rho-U \rho-U_{T} \rho .
$$

For the convenience of the next variation, we rewrite expression (??) in the explicit form in terms of the random functions $\theta$ and $\rho$ :

$$
\begin{array}{r}
\tilde{\mathcal{L}}_{T}(\rho, \theta)=-\hbar \frac{\partial \theta}{\partial t} \rho-\left\{\frac{\hbar^{2}}{2 m}\left(\frac{\partial \theta}{\partial q}\right)^{2} \rho-\frac{\hbar^{2}}{2 m} \Upsilon \frac{\partial \theta}{\partial q} \frac{\partial \rho}{\partial q}+\frac{\hbar^{2}}{8 m} \Upsilon^{2} \frac{1}{\rho}\left(\frac{\partial \rho}{\partial q}\right)^{2}\right\}- \\
-U \rho-\frac{\hbar^{2}}{8 m} \alpha^{2} \frac{1}{\rho}\left(\frac{\partial \rho}{\partial q}\right)^{2}
\end{array}
$$


Varying the action $\mathcal{S}$ with $\widetilde{\mathcal{L}}_{T}$ of form (??) with respect to $\theta$ leads again to the FokkerPlanck equation, which is analogous to (??), but with the replacement of $D_{q u}$ with the effective diffusion coefficient $\mathbb{D}$ in it:

$$
\frac{\partial \rho}{\partial t}+\frac{\partial}{\partial q}\left(\rho \frac{\hbar}{m} \frac{\partial \theta}{\partial q}\right)-\mathbb{D} \frac{\partial^{2} \rho}{\partial q^{2}}=0
$$

Accordingly, varying $\mathcal{S}$ with respect to $\rho$ leads to the Hamilton-Yacobi equation generalized to the case of the stochastic influence of the "thermal vacuum":

$$
\begin{aligned}
\hbar \frac{\partial \theta}{\partial t}+\frac{\hbar^{2}}{2 m}\left(\frac{\partial \theta}{\partial q}\right)^{2}+\frac{\hbar^{2}}{2 m} \Upsilon \frac{\partial^{2} \theta}{\partial q^{2}} & +U(q)- \\
& -\frac{\hbar^{2}}{8 m} \Xi_{T}\left[\frac{1}{\rho^{2}}\left(\frac{\partial \rho}{\partial q}\right)^{2}+2 \frac{\partial}{\partial q}\left(\frac{1}{\rho} \frac{\partial \rho}{\partial q}\right)\right]=0
\end{aligned}
$$

where the notation

$$
\Xi_{T}=2 \Upsilon^{2}-1=2 \operatorname{coth}^{2} \frac{\varkappa \omega}{T}-1,\left.\quad \Xi_{T}\right|_{T=0} \equiv \Xi_{0}=1
$$

was introduced for convenience.

In turn, obtained equations (??) and (??) generalize Eqs. (??) and (??), which allows consistently taking the stochastic influence of the warm vacuum into account. It is indirectly represented in the quantities $\mathbb{D}, \Xi_{T}$, and $\Upsilon$, which are contained in these equations and are dependent on the world constants $\hbar$ and $k_{B}$. This means physically that both types of stochastic environmental influence are taken into account simultaneously: the quantum one characterized by the Planck constant $\hbar$ and the thermal one characterized by the Boltzmann constant $k_{B}$.

Of course, the set of the Fokker-Planck equations (??) and Hamilton-Yacobi equations (??) is a nontrivial generalization of the Schrödinger equation. There are two ways of using them later on. This system can be directly solved for unknown dissimilar functions $\rho$ and $\theta$. As we showed in [?] recently, this allows obtaining nonequilibrium wave functions whose amplitudes and phases are temperature-dependent, and macroparameters in nonequilibrium states can be calculated using them. But these equations can also be modified by endowing them with the form of equations of two-velocity stochastic hydrodynamics for the characteristic velocities $v$ and $u$. As we will see, these equations are a generalization of the corresponding equations of the Nelson stochastic mechanics.

\section{One-dimensional model of two-velocity stochastic hydrodynamics}

To modify the system of equations (??) and (??), we can make the next step and endow these equations with the form of equations for variables of the same type, namely, the velocities $v$ and $u_{e f}$, which are typical of any Markovian processes. In this case, we obtain the system of equations of two-velocity stochastic hydrodynamics generalizing the equations of Nelson stochastic mechanics to the case of the quantum-thermal environmental influence. 
We now show that Eqs. (??) and (??) really allow obtaining equations of stochastic hydrodynamics in the most convenient form. Taking the preceding into account, we are only dealing with the one-dimensional model. To perform the corresponding transformation, we first endow continuity equation (??) with the form of the equation for the diffusion velocity, which, in accordance with (??), can be written in the form

$$
u_{e f}=-\mathbb{D} \frac{\partial \ln \rho}{\partial q}
$$

To do this, we first transform Eq. (??) by introducing $v$ and $u_{e f}$ explicitly in it and then multiply it by $(-\mathbb{D} / \rho)$ :

$$
-\frac{\mathbb{D}}{\rho} \cdot \frac{\partial \rho}{\partial t}-\frac{\mathbb{D}}{\rho}\left[\rho \frac{\partial\left(v+u_{e f}\right)}{\partial q}+\frac{\partial \rho}{\partial q}\left(v+u_{e f}\right)\right]=0 .
$$

We next differentiate the result with respect to $q$, change the order of differentiation in the first term, and form (where it is possible) $\log \rho$, which allows introducing $u_{e f}$ everywhere. As a result, we obtain

$$
\frac{\partial u_{e f}}{\partial t}+\frac{\partial}{\partial q}\left(v u_{e f}\right)+\frac{\partial}{\partial q} u_{e f}^{2}-\mathbb{D} \frac{\partial^{2}}{\partial q^{2}}\left(v+u_{e f}\right)=0 .
$$

It can be endowed with a more elegant form by forming the substantial derivative of the diffusion velocity $u_{e f}$, which is typical of hydrodynamics:

$$
\frac{d u_{e f}}{d t} \equiv \frac{\partial u_{e f}}{\partial t}+u_{e f} \frac{\partial u_{e f}}{\partial q}=-\frac{\partial}{\partial q}\left(v u_{e f}\right)-\frac{\partial}{\partial q} \frac{u_{e f}^{2}}{2}+\mathbb{D}_{e f} \frac{\partial^{2}}{\partial q^{2}}\left(v+u_{e f}\right) .
$$

To endow Eq. (??) with the explicit hydrodynamic form, we also rewrite it in the variables $v$ and $u_{e f}$ :

$$
\hbar \frac{\partial \theta}{\partial t}+\frac{m}{2} v^{2}+\frac{\hbar}{2} \Upsilon \frac{\partial v}{\partial q}+U(q)-\frac{m}{2} \Xi_{T} \cdot\left(u_{e f}^{2}-\frac{\hbar}{m} \frac{\partial u_{e f}}{\partial q}\right)=0 .
$$

To exclude the function $\theta$, we differentiate Eq. (??) with respect to $q$, change the order of differentiation in the first term, and introduce the function $v$ explicitly in it in accordance with (??). As a result, we obtain

$$
\frac{d v}{d t} \equiv\left(\frac{\partial v}{\partial t}+v \frac{\partial v}{\partial q}\right)=-\frac{1}{m} \frac{\partial U}{\partial q}+\Xi_{T} \frac{\partial}{\partial q} \frac{u_{e f}^{2}}{2}-\frac{\hbar}{2 m}\left(\Xi_{T} \frac{\partial^{2} u_{e f}}{\partial q^{2}}+\Upsilon \frac{\partial v^{2}}{\partial q^{2}}\right)
$$

by forming the substantial derivative of the drift velocity $v$ in it too.

We recall that, as the velocity $v$, these equations contain only the quantity $\Delta v$ generated by the stochastic influence. In the case under consideration, as in the case $T=0$, the expressions for $\rho$ and $\theta$ are related to the wave functions of thermal correlated-coherent states, in which the exponent of the exponential depends on $q^{2}$ [?]. It hence follows that 
the last terms in Eqs. (??) and (??) containing the second derivatives of $v \equiv \Delta v$ and $u_{e f}$ with respect to $q$ vanish.

As a result, in the general case, the system of equations for the one-dimensional model of two-velocity stochastic hydrodynamics becomes

$$
\left\{\begin{array}{l}
\frac{d u_{e f}}{d t} \equiv \frac{\partial u_{e f}}{\partial t}+\frac{\partial}{\partial q} \frac{u_{e f}^{2}}{2}=-\frac{\partial}{\partial q}\left(v u_{e f}\right)-\frac{\partial}{\partial q} \frac{u_{e f}^{2}}{2} \\
\frac{d v}{d t}=-\frac{1}{m} \frac{\partial U}{\partial q}+\Xi_{T} \frac{\partial}{\partial q} \frac{u_{e f}^{2}}{2} .
\end{array}\right.
$$

We note that, in the general case, proposed equations (??) are valid for any temperature.

Both equations of this system take into account self-diffusion in the warm vacuum, which is characterized by the coefficient $D_{e f}$ contained in $u_{e f}$. In addition, the right-hand side of the lower equation of the system contains the gradient of diffusion pressure energy density reflecting the stochastic quantum-thermostat influence, including the case $T=0$, in addition to the gradient of classical potential $U(q)$. The analogous contribution of the diffusion pressure energy of the quantum thermostat is also contained in the right-hand side of the upper equation of (??); in this case, it does not vanish even for $v=0$.

For the comparison with the equations of the Nelson stochastic mechanics

$$
\left\{\begin{array}{l}
\frac{\partial u}{\partial t}=-\frac{\partial}{\partial q}(v u), \\
\frac{d v}{d t}=-\frac{1}{m} \frac{\partial U}{\partial q}+\frac{\partial}{\partial q} \frac{u^{2}}{2},
\end{array}\right.
$$

which are valid only at $T=0$, we consider system of equations (??) in the case of the cold vacuum $(T=0)$.

In this case, $u_{e f}$ transforms into $u$. In addition, for the convenience of comparison, in the upper equation of this system, we return to the partial derivative with respect to the time; to do this, we combine similar terms in formula (??). As a result, we have

$$
\left\{\begin{array}{l}
\frac{\partial u}{\partial t}=-\frac{\partial}{\partial q}(v u)-\frac{\partial}{\partial q} u^{2} \\
\frac{d v}{d t}=-\frac{1}{m} \frac{\partial U}{\partial q}+\frac{\partial}{\partial q} \frac{u^{2}}{2}
\end{array}\right.
$$

As is seen, the lower equations of systems (??) and (??) are identical completely. However, these systems of equations differ significantly, which is noticeable when comparing the upper equations. As was expected, it is related to the fact that our theory takes into account self-diffusion occurring even in the cold vacuum. As a consequence of this, the equation for the diffusion velocity contains the gradient of diffusion pressure energy density.

Below, we compare the solutions of system of equations (??) and (??) to establish important physical distinctions between them occurring at $T=0$. In fact, it is interesting to know how the inclusion of the self-diffusion in the "cold vacuum" $(T=0)$ in Eqs. (??) affects the forms of the obtained solutions compared with those of system (??). 
7. Study of the solutions of Eqs. (??) and (??)

We note that, as shown in Sec. 6, system (??) was obtained by taking self-diffusion at arbitrary temperatures into account, while system (??) is valid only at $T=0$. Therefore, only system of equations (??), which is a particular case of (??) at $T=0$, and system (??) can be compared correctly.

\subsection{Apparatus for the determination of the class of equations}

As is known, the total derivative is given by

$$
\frac{d v}{d t}=\frac{\partial v}{\partial q} \frac{\partial q}{\partial t}+\frac{\partial v}{\partial t}=v \frac{\partial v}{\partial q}+\frac{\partial v}{\partial t}
$$

Now we rewrite Eqs. (??) and (??) completely in the partial derivatives; for convenience, we set $\frac{1}{m} \frac{\partial U}{\partial q}=\widetilde{\alpha}(q)$. Then systems of equations (??) and (??) become

$$
\begin{aligned}
& \frac{\partial u}{\partial t}+v \frac{\partial u}{\partial q}+u \frac{\partial v}{\partial q}=0 \\
& \frac{\partial v}{\partial t}+v \frac{\partial v}{\partial q}-u \frac{\partial u}{\partial q}=\widetilde{\alpha}(q)
\end{aligned}
$$

respectively, and

$$
\begin{aligned}
& \frac{\partial u}{\partial t}+(v+2 u) \frac{\partial u}{\partial q}+u \frac{\partial v}{\partial q}=0 \\
& \frac{\partial v}{\partial t}+v \frac{\partial v}{\partial q}-u \frac{\partial u}{\partial q}=\widetilde{\alpha}(q)
\end{aligned}
$$

where $u$ is the diffusion velocity, $v$ is the drift velocity. Equations (??) and (??) are quasilinear systems of differential equations of first order for two unknown functions $u(t, q)$ and $v(t, q)$ of two variables. We note that the main distinction of system (??) from system (??) is that the assumption of self-diffusion was used when deriving the latter.

In this paper, we study homogeneous systems (??) (??) and set $\widetilde{\alpha}(q)=0$. The establishing of the type of equation (elliptic, hyperbolic, or parabolic one) is the most important fact determining the solution of these equations. As is known, to solve hyperbolic equations, the concept of characteristic (integrals of a certain characteristic equation) is used. The elliptic operator has no characteristics in the real domain, and, in general, stationary equilibrium states correspond to elliptic differential equations in physics. Thus, the establishing of the class to which the corresponding system belongs allows drawing the conclusion about the character of solutions of the equation and set them into correspondence with a certain physical interpretation.

Starting from the foregoing, we begin our study. Equations (??) and (??) are system of quasilinear partial differential equations of first order for two unknown functions. 
Therefore, following [?], we represent each of the equations in systems (??) and (??) in the form

$$
\begin{aligned}
& L_{1}=A_{1} u_{t}+B_{1} u_{q}+C_{1} v_{t}+D_{1} v_{q}, \\
& L_{2}=A_{2} u_{t}+B_{2} u_{q}+C_{2} v_{t}+D_{2} v_{q},
\end{aligned}
$$

where $A_{i}, B_{i}, C_{i}, D_{i}$ are known functions of the variables $t, q, u, v, i=1,2$. We assume that all the considered functions are continuous and have continuous derivatives of required order. It is well known that the linear combination $a f_{x}+b f_{y}$ of partial derivatives of the function of two variables $f(x, y)$ is the derivative in the direction specified by the relations $\frac{d x}{d y}=\frac{a}{b}$. If $x(l), y(l)$ is a curve with $\frac{x_{l}}{y_{l}}=\frac{a}{b}$, then $a f_{x}+b f_{y}$ is the derivative of the function $f$ along this curve. This fact allows us to elegantly pass from systems (??) and (??) of partial differential equations to the study of algebraic equations.

We consider functions $u(t, q), v(t, q)$ for which the coefficients in differential equations (??) depend only on $t$ and $q$. We find the linear combination

$$
L=\lambda_{1} L_{1}+\lambda_{2} L_{2},
$$

such that the differential expression $L$ contains the derivatives only along one direction. Such a direction that depends on the point $(t, q)$ and on the functions $u(t, q)$ and $v(t, q)$ at this point is called characteristic. Let this direction be specified by the ratio $t_{l}: q_{l}$. As was mentioned above, the condition that the function $u(t, q), v(t, q)$ in the differential expression $L$ are differentiated in this direction then looks as follows

$$
\frac{\lambda_{1} A_{1}+\lambda_{2} A_{2}}{\lambda_{1} B_{1}+\lambda_{2} B_{2}}=\frac{\lambda_{1} C_{1}+\lambda_{2} C_{2}}{\lambda_{1} D_{1}+\lambda_{2} D_{2}}=\frac{t_{l}}{q_{l}},
$$

because the coefficients at the derivatives $u_{t}, u_{q}$ and $v_{t}, v_{q}$ in the expression $L$ are determined by the corresponding terms in proportions (??). Multiplying expression (??) by $t_{l}$, we obtain

$$
\begin{aligned}
& L t_{l}=\left(\lambda_{1} A_{1}+\lambda_{2} A_{2}\right) u_{t} t_{l}+\left(\lambda_{1} B_{1}+\lambda_{2} B_{2}\right) u_{q} t_{l}+ \\
& \left(\lambda_{1} C_{1}+\lambda_{2} C_{2}\right) v_{t} t_{l}+\left(\lambda_{1} D_{1}+\lambda_{2} D_{2}\right) v_{q} t_{l}= \\
& \left(\lambda_{1} A_{1}+\lambda_{2} A_{2}\right)\left(u_{l}-u_{q} q_{l}\right)+\left(\lambda_{1} B_{1}+\lambda_{2} B_{2}\right) u_{q} t_{l}+ \\
& \left(\lambda_{1} C_{1}+\lambda_{1} C_{2}\right)\left(v_{l}-v_{q} q_{l}\right)+\left(\lambda_{1} D_{1}+\lambda_{2} D_{2}\right) v_{q} t_{l}= \\
& \left(\lambda_{1} A_{1}+\lambda_{2} A_{2}\right) u_{l}+\left(\lambda_{1} C_{1}+\lambda_{2} C_{2}\right) v_{l}- \\
& {\left[\left(\lambda_{1} A_{1}+\lambda_{2} A_{2}\right) q_{l}-\left(\lambda_{1} B_{1}+\lambda_{2} B_{2}\right) t_{l}\right] u_{q}-} \\
& {\left[\left(\lambda_{1} C_{1}+\lambda_{2} C_{2}\right) q_{l}-\left(\lambda_{1} D_{1}+\lambda_{2} D_{2}\right) t_{l}\right] v_{q}=} \\
& \quad\left(\lambda_{1} A_{1}+\lambda_{2} A_{2}\right) u_{l}+\left(\lambda_{1} C_{1}+\lambda_{2} C_{2}\right) v_{l},
\end{aligned}
$$

because, in view of (??), we have

$$
\begin{aligned}
\left(\lambda_{1} A_{1}+\lambda_{2} A_{2}\right) q_{l}-\left(\lambda_{1} B_{1}+\lambda_{2} B_{2}\right) t_{l}= & \\
& \left(\lambda_{1} C_{1}+\lambda_{2} C_{2}\right) q_{l}-\left(\lambda_{1} D_{1}+\lambda_{2} D_{2}\right) t_{l}=0 .
\end{aligned}
$$


Analogously, multiplying $L$ by $q_{l}$, we obtain

$$
L q_{l}=\left(\lambda_{1} B_{1}+\lambda_{2} B_{2}\right) u_{l}+\left(\lambda_{1} D_{1}+\lambda_{2} D_{2}\right) v_{l} .
$$

If the functions $u(t, q), v(t, q)$ are solutions of system (??), and the expression $L$ has the derivative in the direction $l$, which is given by the ratio $t_{l}: q_{l}$, then, from (??), it is easy to obtain the system of two linear homogeneous algebraic equations for $\lambda_{1}$ and $\lambda_{2}$

$$
\begin{aligned}
& \lambda_{1}\left(A_{1} q_{l}-B_{1} t_{l}\right)+\lambda_{2}\left(A_{2} q_{l}-B_{2} t_{l}\right)=0, \\
& \lambda_{1}\left(C_{1} q_{l}-D_{1} t_{l}\right)+\lambda_{2}\left(C_{2} q_{l}-D_{2} t_{l}\right)=0 .
\end{aligned}
$$

System (??) has a nontrivial solution if it has the determinant that is equal to zero, i.e.,

$$
\left|\begin{array}{ll}
A_{1} q_{l}-B_{1} t_{l} & A_{2} q_{l}-B_{2} t_{l} \\
C_{1} q_{l}-D_{1} t_{l} & C_{2} q_{l}-D_{2} t_{l}
\end{array}\right|=0
$$

which is convenient to write in the quadratic form

$$
a t_{l}^{2}-2 b t_{l} q_{l}+c q_{l}^{2}=0
$$

where $a=[B D], 2 b=[A D]+[B C], c=[A C],[X Y]=X_{1} Y_{2}-X_{2} Y_{1}$.

Depending on the sign of the determinant of form (??), it is possible to classify the equations as follows.

1. If $b^{2}-a c<0$, then quadratic form (??) is nonzero for any real $t_{l}, q_{l}$; and, consequently, there is no real characteristic direction, and the system of differential equations belongs to the elliptic type.

2. If $b^{2}-a c>0$, then two characteristic directions specified by the ratio $t_{l}: q_{l}$ exist at each point; they correspond to two different roots $\lambda_{1}$ and $\lambda_{2}$ of the quadratic form (??). In this case, the system of differential equations belongs to the hyperbolic type.

3. In the case where $b^{2}-a c=0$, expression (??) has one root of multiplicity 2 , and there is one degenerate direction corresponding to this root; the system of differential equations belongs to the parabolic type.

\subsection{Study of Eqs. (??) and (??)}

Starting from the foregoing, we analyze the class of system of Nelson equations (??). For this system, the coefficients have the form

$$
\begin{aligned}
& A_{1}=1, B_{1}=v, C_{1}=0, D_{1}=u, \\
& A_{2}=0, B_{2}=-u, C_{2}=1, D_{2}=v .
\end{aligned}
$$

It hence follows that

$$
b^{2}-a c=-u^{2}<0
$$


Thus, the hydrodynamic system of Nelson equations is elliptic and cannot be used to study the fluctuation evolution in the quantum-mechanical description of the system.

For system (??), the coefficients have another form

$$
\begin{aligned}
& A_{1}=1, B_{1}=2 u+v, C_{1}=0, D_{1}=u, \\
& A_{2}=0, B_{2}=-u, C_{2}=1, D_{2}=v,
\end{aligned}
$$

which leads to

$$
b^{2}-a c=0
$$

The latter demonstrates that system of equations (??) is parabolic, i.e., the system of the evolution type and, consequently, can be used to describe the evolution of perturbations appearing in the case of fluctuations.

We find the characteristic direction for system (??). In accordance with the foregoing, we form the linear combination of two equations of this system and require that it contain the derivatives of the functions $u(q, t)$ and $v(q, t)$ only in one direction $u_{l}, v_{l}$ that is given by $\left(t_{l}, q_{l}\right)$ :

$$
L=u_{t}+(2 u+v-\lambda u) u_{q}+\lambda v_{t}+(u+\lambda v) v_{q}=0 .
$$

Then, in accordance with (??), the conditions determining the direction $\left(t_{l}, q_{l}\right)$ looks as follows:

$$
\begin{aligned}
q_{l} & =(2 u+v-\lambda u) t_{l}, \\
\lambda q_{l} & =(u+\lambda v) t_{l},
\end{aligned}
$$

whence

$$
(\lambda-1)^{2}=0 .
$$

Consequently, in problem (??), there is one characteristic direction determined by the condition

$$
q_{l}=(u+v) t_{l} .
$$

From (??) - (??), we obtain the characteristic equation for $u$ and $v$

$$
u_{l}+v_{l}=0 \text {. }
$$

The meaning of Eq. (??) is that the characteristic in the $(q, t)$ plane represents the motion of possible perturbations whose velocity

$$
\frac{d q}{d t}=u+v
$$

is the sum of the drift and diffusion velocities.

In the context of the use of this system, we keep in mind the following fact. The fluctuations of parameters, for example, of the temperature, the density, and the pressure, necessarily produce perturbations of the variables $u$ and $v$ in the hydrodynamic equations. The evolution of these perturbations can be described and studied using system of equations (??). 
7.3. Numerical simulation of the solutions of Eqs. (??) and (??).

Our above considerations are illustrated using the numerical simulation of the solution of systems (??) and (??). The model equation for these systems is the transport equation written in the vector form:

$$
\frac{\partial y}{\partial t}+A(y) \frac{\partial y}{\partial q}=f
$$

where $y=(u, v)^{\mathrm{T}}, A \in \mathbb{R}^{2 \ltimes 2}$ is the system matrix, and $f \in \mathbb{R}^{2}$. For systems (??) and (??), we consider the Cauchy problem

$$
\begin{aligned}
& y(q, 0)=y_{0}(q), \\
& q \in \mathbb{R}, \quad t>0,
\end{aligned}
$$

on the real axis. To solve the problem in the $(q, t)$ plane, we use the mesh

$$
\begin{aligned}
\omega_{h \tau} & =\omega_{h} \times \omega_{\tau}, \\
\omega_{h} & =\left\{q_{k}=k h, k=0, \pm 1, \pm 2, \ldots\right\}, \\
\omega_{\tau} & =\left\{t_{n}=n \tau, \quad n=0,1,2, \ldots\right\},
\end{aligned}
$$

with the step $h$ with respect to $q$ and with the step $\tau$ with respect to $t$. The solution of the problem was studied using the implicit three-layer scheme

$$
\frac{3 y_{k}^{n+1}-4 y_{k}^{n}+y_{k}^{n-1}}{2 \tau}+A(y) \frac{y_{k+1}^{n+1}-y_{k-1}^{n+1}}{2 h}=\varphi,
$$

with the approximation order $O\left(\tau^{2}+h^{2}\right)$. Problem (??) was solved by the iteration method. We show that scheme (??) is absolutely stable. For the homogeneous scalar equation

$$
\frac{\partial y}{\partial t}+a(y) \frac{\partial y}{\partial q}=0
$$

we seek a solution of problem (??) in the form

$$
y_{k}^{n}=\eta^{n} e^{i k h \theta},
$$

where $i=\sqrt{-1}, \theta \in \mathbb{R}$. Substituting (??) in the equation

$$
\frac{3 y_{k}^{n+1}-4 y_{k}^{n}+y_{k}^{n-1}}{2 \tau}+a(y) \frac{y_{k+1}^{n+1}-y_{k-1}^{n+1}}{2 h}=0,
$$

after simple transformations, we obtain the equation for $\eta$

$$
\mu \eta^{2}-4 \eta+1=0
$$

where $\mu=3+2 a \gamma i \sin \theta, \gamma=\frac{\tau}{h}$. 
We find the ensemble of points $G$ of the complex plane $\mu=r+i s$ for which the absolute values of the roots of Eq. (??) do not exceed unity. The boundary of the domain $G$ is the set of points $\mu$ for which $|\eta|<1$. We express the parameter $\mu$ in Eq. (??) in terms of the variable $\eta$

$$
\mu=\frac{4}{\eta}-\frac{1}{\eta^{2}}
$$

It is obvious that if $|\eta|=1$, then, setting $\eta=e^{-i \varphi}$, we obtain

$$
\mu=4 e^{i \varphi}-e^{2 i \varphi} .
$$

If the argument $\varphi$ is varied from 0 to $2 \pi$, then the points $\mu$ describe the closed curve $\Gamma$; it is convenient to represent the equation of this curve in the plane $\mu=r+i s$ in the parametric form

$$
\begin{array}{r}
r=4 \cos \varphi-\cos 2 \varphi, \\
s=4 \sin \varphi-\sin 2 \varphi .
\end{array}
$$

It can be seen from (??) that the curve $\Gamma$ is symmetric with respect to the real axis $r$. It intersects the axis $r$ at the points $\mu(0)=3$ and $\mu(\pi)=-5$. At these points, the derivatives

$$
\frac{d s}{d r}=\frac{2 \cos \varphi-\cos 2 \varphi}{\sin 2 \varphi-2 \sin \varphi}, \quad \frac{d^{2} s}{d r^{2}}=\frac{-3}{4 \sin \varphi(1-\cos \varphi)^{2}}
$$

are not determined. In this case, the second derivative of the curve $\Gamma$ is negative for $0<\varphi<\pi$ and positive for $\pi<\varphi<2 \pi$. This is evidence of the fact that the closed curve $\Gamma$ is convex upward in the upper half-plane $\mu$ and convex downward in the lower half-plane. Consequently, the domain inside the closed curve $\Gamma$ is convex (Fig. ??). In this case, the straight line $\mu=3+i$ s touches the curve $\Gamma$ at the point $\mu=3$. The other points of this straight line lie in the domain located outside this curve $\Gamma$. We show that the condition $|\eta|<1$ is satisfied in this domain, and the set of points lying outside the curve $\Gamma$ is the stability domain of scheme (??).

Indeed, we consider the solution of the equation

$$
(3+i s) \eta^{2}-4 \eta+1=0
$$

for $0<s<1$. Then one of the roots

$$
\eta=\frac{2+\sqrt{1-i s}}{3+i s}
$$

corresponding to the maximum value of the modulus $|\eta|$ can be written in the form

$$
\eta=\frac{9-\frac{s^{2}}{2}-\frac{9 i s}{2}}{9+s^{2}}+O(s) .
$$




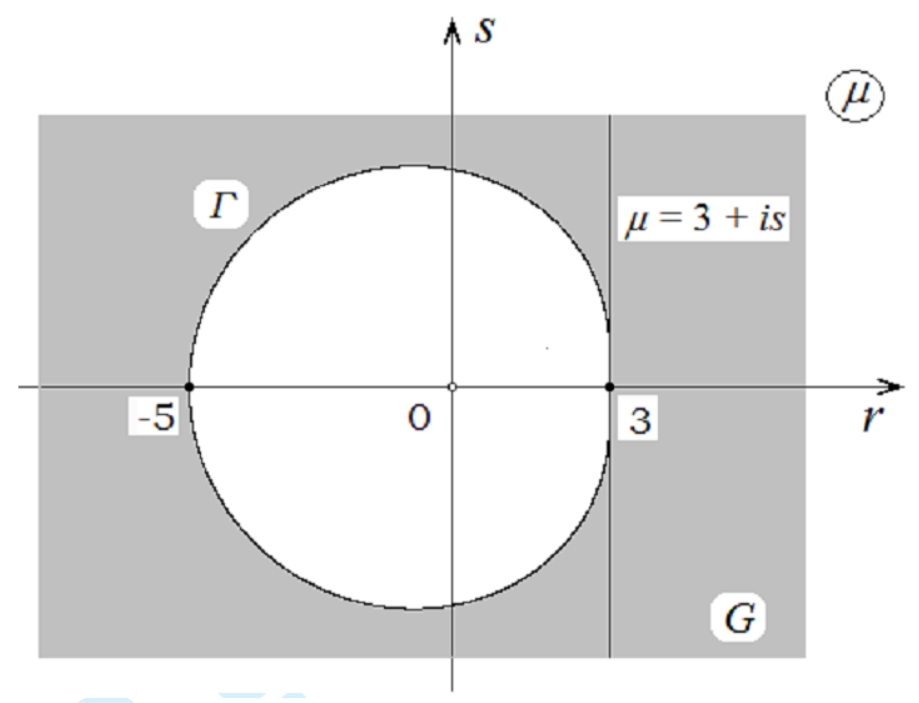

Figure 1: The border of stability of scheme (??).

The modulus of this value is

$$
|\eta|=\frac{\sqrt{81+\frac{45 s^{2}+s^{4}}{4}}}{9+s^{2}}=\sqrt{1-\frac{27 s^{2}+s^{4}}{4\left(9+s^{2}\right)}}=1-O\left(s^{2}\right)<1 .
$$

Thus, the set of points $\mu=3+2 a \gamma i \sin \theta$ lies completely in the stability domain, where $|\eta| \leq 1$. Consequently, scheme (??) is absolutely stable and is independent of the quantity $\gamma=\frac{\tau}{h}$ as it shown in Fig. ??. In the numerical simulation, we studied the solution for a certain perturbation given in the initial condition in a neighborhood of the point $q=0$, which is one of the variables of the initial homogeneous condition. This perturbation in the initial condition models the result of the fluctuation of the physicalsystem macroparameter. The calculations carried out numerically using the proposed implicit scheme showed the following result. Any (even arbitrarily small) perturbation in the system of Nelson equations leads to an unbound growth of the variables $u$ and $v$. This is a consequence of the fact that the Nelson equations belong to the elliptic type and can be used to describe stationary processes such as flow over bodies by a flux, problems of electrostatics, stationary problems of gravitation theory.

When solving system of equations (??), we obtained the solution of the form of running perturbation wave with respect to the spatial coordinate. In this case, the evolution of the perturbation itself is observed along with the perturbation displacement. Figure shows the numerical solutions of system (??) for different instants of time: a) $t=0, \quad$ b) $t=\tau$, c) $t=20 \tau$, d) $t=50 \tau$, where $\tau$ is the integration step with respect to the time. 


\subsection{Discussion of the results of numerical simulation}

When stating the problem of numerical simulation, we start from the fact the fluctuations of macroparameters of the physical system (temperature, density, and pressure) must produce perturbations of the drift $v$ and the diffusion $u$ velocities in the hydrodynamic equations. Therefore, we intended to comparatively analyze the behavior of the small perturbation of the quantities $v$ and $u$ as the final aim of the numerical study of system of Nelson hydrodynamic equations (??) and system (??) obtained in this paper. In other words, our problem was to clarify to what extent each of these systems of equations is appropriate for describing the dynamics of quantum-thermal fluctuations. To answer this question, a weak perturbation with respect to the variable $u$ in a neighborhood of the point $q_{0}$ was introduced as an initial condition in systems of equations (??) and (??), which are most convenient for the analysis. The numerical calculations carried out using the above-mentioned implicit scheme showed the following.

1. In the Nelson system of equations, any, even an arbitrarily small perturbation leads to an unbound increase in the variables $u$ and $v$. This is a consequence of the fact that the Nelson equations are of the elliptic type and are intended to describe stationary processes such as a flow around bodies, electrostatic problems, stationary problems of gravitation theory, etc.

2. On the contrary, system of equations (??) demonstrated a quite different result, namely, the fluctuation evolutions in time and space, as shown in Fig. ??.

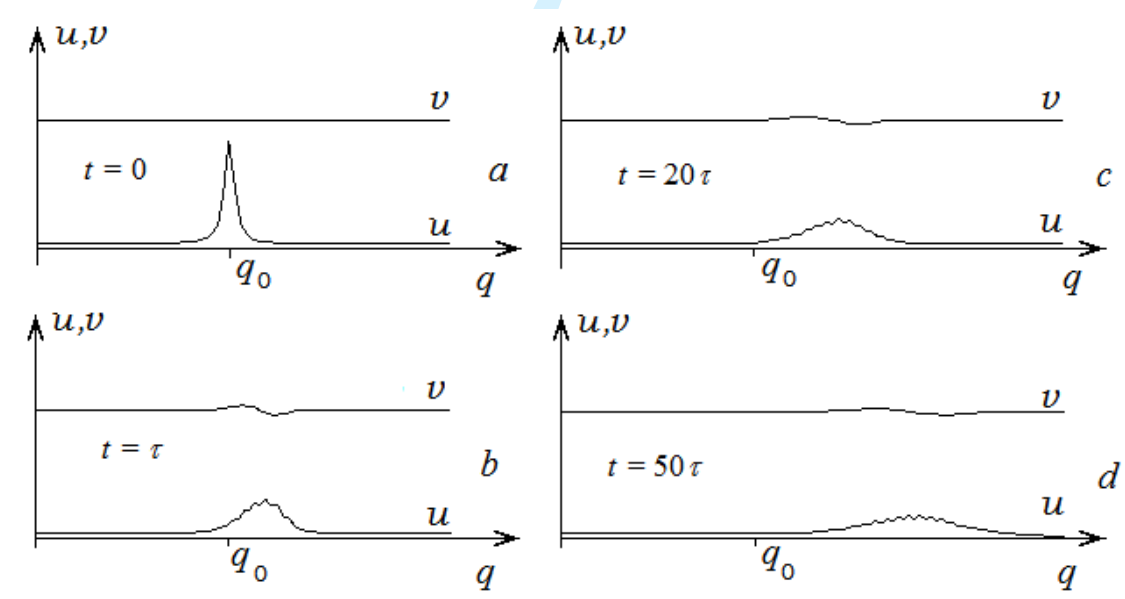

Figure 2: Results of numerical solution of system (??).

The initial conditions of the solution of problem (??) at the instant of time $t=0$ are given in Fig. ??a. The behavior of the variables $u$ and $v$ at the subsequent instants are shown in Figs. ??b- ??d. It follows from them that the initial perturbation with respect to $\mathrm{u}$ decreases very rapidly. Its amplitude decreases significantly even during the period $t=\tau$, and, moreover, it does not remain localized at the point $q_{0}$ and is dispersed over the space, moving along the coordinate $q$ (Fig. ??b). In this case, the response to the perturbation of the diffusion velocity $u$ also affects the drift velocity $v$. We note that its 
graph is two-hump. This reflects the fact that diffusion occurs along the flow and in the opposite direction.

Figures ??c and ??d shows the solutions of system (??); they demonstrate the perturbation evolution at the subsequent instants of time $t=20 \tau$ and $t=50 \tau$, respectively. This evolution is manifested by the further decrease in the amplitudes of the perturbation with respect to both variables $u$ and $v$, in the spread of perturbations over the space with their simultaneous movement in the space, which is caused by the drift velocity. It can be interpreted as a continuation of the decrease in the amplitude and the further motion of the weakened perturbation caused by the drift velocity.

\section{Conclusion}

Fenyes [?] was, probably, the first to put forward the idea of using the Lagrangian density $\mathcal{L}[\rho ; \theta]$ in quantum theory. The Fokker-Planck equation containing the total probability flux velocity $V$ with the diffusion coefficient either $D_{q u}$ or $D_{T}$ followed from the expression proposed by him. However, he did not introduce the generalized diffusion coefficient $D_{e f}$. We note that he obtained another equation of motion of the Hamilton-Jacobi type in this paper. Moreover, he there stated that the obtained system of equations for the functions $\rho$ and $\theta$ is completely equivalent to the Schrödinger equations for the functions $\psi$ and $\psi^{*}$, in spite of the fact that, unlike the Schrödinger equation, the Fokker-Planck equation leads to the irreversibility.

Unlike [?], in our approach, we have consistently taken into account quantum-thermal fluctuations and the density of diffusion pressure energy related to the stochastic influence of the environment (the quantum thermostat) for $T \geqslant 0$. As a final result, we endowed the Fokker-Planck and Hamilton-Jacobi equations with the form of system of equations (??) for the one-dimensional model of two-velocity stochastic hydrodynamics. The selfdiffusion coefficient in them is determined by the effective environmental influence that is dependent on the funcdamental constant $\varkappa=\frac{\hbar}{2 k_{B}}$.

In our opinion, on this way, in the future, it is possible to construct the full-scale stochastic hydrodynamics taking into account not only self-diffusion but also shear viscosity and then use it to describe interesting media such as nearly perfect fluids (NPF). To do this, it is necessary to pass from the lower equation in (??) for the drift velocity to the equation that is a generalization of the Navier-Stokes equation to the case in which self-diffusion is taken into account.

It follows from our analysis that the self-diffusion coefficient $D_{\text {ef }}=\mathbb{J} / m$ is, probably, the most adequate characteristic of transport phenomena and is important for describing dissipative processes. To-day, it is possible to determine it experimentally by studying the diffusion of massive quarks in the quark-gluon plasma obtained during the collision between heavy ions.

The numerical analysis of the behavior of the solutions of the particular case of system (??) in form (??), which is valid at $T=0$, showed that these equations illustrate the perturbation relaxation. Thus, self-diffusion can be regarded as a hydrodynamic mechanism 
for the relaxation of quantum-thermal fluctuations. We intend to study the behavior of the solution of system (??) in the general case later on.

Thus, we suggest that the hydrodynamic approach to quantum theory proposed in this paper allows, in principle, studying quantum-thermal fluctuations by means of the obtained hydrodynamic equations.

We are grateful to N.F.Shul'ga, I.M. Mrygloda, A.G. Zagorodnii, I.V. Volovich, N.M. Plakida, Yu.P. Rybakov, and also the participants of scientific seminars guided by them in Kiev, Kharkov, Samara, and Moscow for the fruitful discussion of the results presented above.

We must say out of the sence of duty that the initial plan, the problem statement, and the key ideas were proposed by our teacher and co-author A.D. Sukhanov to the blessed memory of whom this paper is dedicated.

\section{References}

[1] D. Forster. Hydrodynamics Ffluctuations, Broken Symmetry, and Correlation Functions. Persens Books, 1990, 326 p.

[2] A.D. Sukhanov, O.N. Golubjeva, Baryakhtar.UJP, 2011. V.56 (10) , P.1122

[3] R.Z. Führth. Phys. 1933. V. 81, 143

[4] A.D. Sukhanov. Teor. Mat. Fiz., 2004. V.139, 1 . P.129

[5] E. Nelson. Dynamical theory of Brownian motion. Princeton: Princ. Univ. Press, 1967.

[6] R. Feynman, P. Leiton, M. Sands. Feynman Lectures on Physics V. 9, 1967. P.244

[7] D.I. Blokhintzev. Principles Problems of Quantum Mechanics.(in Russian) M.:Nauka, 1966. P.54

[8] A.N. Kolmogoroff. Math. Ann. 1933 B.104, 415 (1931); ibid B.108,149.

[9] I. Fenyes. Zs. Phys. 1952 B.132. P. 81

[10] O.N. Golubjeva, A.D. Sukhanov. Particles and Nuclei, Letters, 2011. V. 8,1 , P. 1

[11] O.N. Golubjeva, A.D. Sukhanov. CJP, 2014. V.92. P. 259

[12] R. Courant and K.O. Friedrichs. Supersonic Flow and Shock Waves. BerlinHeidelberg-New York. Springer-Verlag, 1976. 